\title{
ON INTEGRAL REPRESENTATION OF VECTOR VALUED MEASURES
}

\author{
ULF RØNNOW
}

\section{Introduction.}

The purpose of the present paper is to establish some general conditions for the existence of integral representations of Banach-valued measures and indicate their relation to the theory of Banach-valued martingales.

The results are based upon two theorems which are stated in Section 1 together with some basic definitions: Theorem A concerns extension of Banach-valued set functions to Banach-valued measures; Theorem B, which was proved by S. D. Chatterji [4, Theorem 1], states a convergence property of a class of Banach-valued martingales. See also [14].

In Section 2, a general condition is given in order that a Banachvalued measure be an integral with respect to a positive measure.

In Section 3, results of R. S. Phillips and S. D. Chatterji, for reflexive Banach spaces, a general Radon-Nikodym property and a convergence property for a certain class of Banach-valued martingales, respectively, are shown to be equivalent for general Banach spaces.

An example is given to show that these properties are not possessed by every Banach space.

Recently, S. D. Chatterji [13, pp. 55-61] has pointed out a similar connection between a general Radon-Nikodym theorem for Banach spaces and a point-wise convergence property of a certain class of Banachvalued martingales.

Finally, we observe that the theory of martingales with values in a topological vector space and Pettis integration is treated in the thesis of M. Metivier [8]. See also A. I. and C. I. Tulcea [14], [15].

\section{Preliminaries.}

In the sequel $\mathscr{X}$ will denote a Banach space. The norm of $x \in \mathscr{X}$ is denoted by $|x|$. A triple $(S, \Sigma, \mu)$ will denote a measure space, i.e. a system which consists of a non-empty set $S$, a $\sigma$-field $\Sigma$ of subsets $S$ such that

Received June 10, 1965; revised October 20, 1966. 
$S \in \Sigma$, and a measure $\mu$, which is assumed to be positive, defined on $\Sigma$.

Set functions. We shall consider set functions whose values are points in a Banach space $\mathscr{X}$, and we shall say that they are $\mathscr{X}$-valued.

Let $\varphi$ be a set function defined on a field $\Gamma$ of subsets of $S$. Then for every $E \in \Gamma$ the total variation of $\varphi$ on $E$, denoted by $v(\varphi, E)$, is defined as

$$
v(\varphi, E)=\sup \sum_{i=1}^{n}\left|\varphi\left(E_{i}\right)\right|,
$$

where the supremum is taken over all finite sequences $\left\{E_{i}\right\}$ of mutually disjoint sets of $\Gamma$ with $E_{i} \cong E$. The set function $\varphi$ is of bounded variation on $E \in \Gamma$ if $v(\varphi, E)<\infty$. A countably additive $\mathscr{X}$-valued set function defined on a $\sigma$-field is called an $\mathscr{X}$-valued measure.

Theorem A. Let $\Gamma$ be a field of subsets of $S$ and let $\Sigma$ be the o-field generated by $\Gamma$.

If $\varphi$ is an additive set function of bounded variation defined on $\Gamma$, then in order that there exist a countably additive extension $\psi$ defined on $\Sigma$ it is necessary and sufficient that $\varphi$ be countably additive.

If the condition is satisfied, the extension is unique and $v(\psi, \cdot)$ is exactly the extension of $v(\varphi, \cdot)$.

A convenient reference to this known theorem is [15, p. 119 and footnote (6)].

Martingales. Let $(S, \Sigma, \mu)$ be a finite measure space and let $Q$ be a subset of the extended natural numbers. Let $\left\{\Sigma_{n}\right\}, n \in Q$, be an increasing sequence of sub- $\sigma$-fields of $\Sigma$. Let $f_{n} \in L_{1}\left(S, \Sigma_{n}, \mu\right)$ or $L_{1}\left(S, \Sigma_{n}, \mu, \mathscr{X}\right)$. Then the system $\left\{f_{n}, \Sigma_{n}, n \in Q\right\}$ is said to constitute a scalar-valued or $\mathscr{X}$-valued martingale, respectively, if

$$
\int_{A} f_{m}(s) \mu(d s)=\int_{A} f_{n}(s) \mu(d s)
$$

for $m, n \in Q, m \leqq n, A \in \Sigma_{m}$.

Theorem B. Let $(S, \Sigma, \mu)$ be a finite measure space, let $\left\{\Sigma_{n}\right\}, 1 \leqq n<\infty$, be an increasing sequence of $\sigma$-fields all contained in $\Sigma$, and let $f \in L_{1}(S, \Sigma, \mu, \mathscr{X})$.

Then there exists a unique $\mathscr{X}$-valued martingale $\left\{f_{n}, \Sigma_{n}, 1 \leqq n \leqq \infty\right\}$, where $\Sigma_{\infty}$ denotes the $\sigma$-field generated by $\left\{\Sigma_{n}\right\}, 1 \leqq n<\infty$, such that

Moreover,

$$
\int_{\boldsymbol{A}} f_{n}(s) \mu(d s)=\int_{\boldsymbol{A}} f(s) \mu(d s), \quad A \in \Sigma_{n}, \quad 1 \leqq n \leqq \infty .
$$


and

$$
\left|f_{1}\right|_{1} \leqq\left|f_{2}\right|_{1} \leqq \cdots \leqq\left|f_{\infty}\right|_{1} \leqq|f|_{1},
$$

$$
\lim _{n \rightarrow \infty}\left|f_{n}-f_{\infty}\right|_{1}=0 \text {. }
$$

The notion of uniform integrability of a family of integrable functions $\left\{f_{t}\right\} \cong L_{1}(S, \Sigma, \mu, \mathscr{X}), t \in T, \mu$ a finite measure, is defined as follows: For every $\varepsilon>0$, there exists a number $\delta>0$ such that

$$
\int_{\left\{\left|f_{t}\right|>\delta\right\}}\left|f_{t}(s)\right| \mu(d s)<\varepsilon \quad \text { for all } t \in T .
$$

\section{Integral representation of $\mathscr{X}$-valued measures.}

The purpose of this section is to give an account of the conditions required in order that an $\mathscr{X}$-valued measure $\varphi$ may be represented as an indefinite integral with respect to a measure $\mu$.

In the sequel we shall use the symbol $\Delta$, with or without suffixes, to denote partitions of $S$ into a finite number of subsets from $\Sigma$ with positive measures.

The set $\{\Delta\}$ of all $\Delta^{\prime}$ 's is partially ordered by inclusion $\bmod \mu$, that is, $\Delta \prec \Delta^{\prime}$ or $\Delta^{\prime} \succ \Delta$ if to each $A \in \Delta^{\prime}$ there exists a set $B \in \Delta$ such that $\mu(A \backslash B)=0$, moreover, the family of $\Delta^{\prime}$ 's is a directed set, since to each finite collection $\left\{\Delta_{i}\right\}$ there always is a $\Delta$ such that $\Delta \succ \Delta_{i}$ for all $\Delta_{i}$.

Defining $f_{\Delta}: S \rightarrow \mathscr{X}$ by

$$
f_{\Delta}(s)=\sum_{A \in \Delta} \frac{\varphi(A)}{\mu(A)} \chi_{\Delta}(s),
$$

the mapping $\Delta \rightarrow f_{\Delta}$ becomes a generalized sequence in $L_{1}(S, \Sigma, \mu, \mathscr{X})$.

Theorem 1. Let $(S, \Sigma, \mu)$ be a finite measure space, and let $\varphi$ be an $\mathscr{X}$-valued set function defined on $\Sigma$.

In order that there exist a function $f \in L_{1}(S, \Sigma, \mu, \mathscr{X})$ such that

$$
\varphi(A)=\int_{A} f(s) \mu(d s), \quad A \in \Sigma,
$$

it is necessary and sufficient that $\varphi$ be additive and that $\left\{f_{\Delta}\right\}$ be a generalized Cauchy sequence in $L_{1}(S, \Sigma, \mu, \mathscr{X})$.

If the condition is satisfied, then $\varphi$ is countably additive, $\mu$-continuous, of bounded variation, and

$$
v(\varphi, A)=\int_{A}|f(s)| \mu(d s), \quad A \in \Sigma .
$$


Proof. First let us assume that

$$
\varphi(A)=\int_{A} f(s) \mu(d s), \quad A \in \Sigma,
$$

where $f \in L_{1}(S, \Sigma, \mu, \mathscr{X})$, and let us prove that $\left\{f_{\Delta}\right\}$ is a generalized Cauchy sequence, i.e. to an arbitrary $\varepsilon>0$ there exists a $\Delta_{\varepsilon}$ such that

$$
\left|f_{\Delta}-f_{\Delta^{\prime}}\right|_{1}<\varepsilon
$$

for any pair $\Delta, \Delta^{\prime}>\Delta_{\varepsilon}$.

If this is not the case, there is an $\varepsilon_{0}>0$ such that there exist for every $\Delta$ two partitions $\Delta^{\prime}$ and $\Delta^{\prime \prime}>\Delta$ for which

$$
\left|f_{\Delta^{\prime \prime}}-f_{\Delta^{\prime}}\right|_{1} \geqq 2 \varepsilon_{0} \text {. }
$$

Accordingly, applying the triangle inequality we obtain

$$
\max \left\{\left|f_{\Delta^{\prime}}-f_{\Delta}\right|_{1},\left|f_{\Delta^{\prime \prime}}-f_{\Delta}\right|_{1}\right\} \geqq \varepsilon_{0} .
$$

Now we can prove by induction the existence of a martingale $\left\{f_{\Delta_{i}}, \Sigma_{i}, 1 \leqq i<\infty\right\}$ for which

$$
\left|f_{\Delta_{i+1}}-f_{\Delta_{i}}\right|_{1} \geqq \varepsilon_{0} \quad \text { for } i=1,2, \ldots .
$$

We proceed as follows: For $i=1$,

$$
\Delta_{1}=\{S\}, \quad f_{\Delta_{1}}=\frac{\varphi(S)}{\mu(S)} \chi_{S}, \quad \text { and } \quad \Sigma_{1}=\{S\} .
$$

For $i=n$ we assume the existence of the sequence

$$
\Delta_{1} \prec \Delta_{2} \prec \ldots \prec \Delta_{n},
$$

such that (3) is satisfied for $i=1,2, \ldots, n-1$. Then there exists by the preceding paragraphs $\Delta^{\prime}, \Delta^{\prime \prime}>\Delta_{n}$ such that (2) is satisfied, and thus $\Delta^{\prime}$ or $\Delta^{\prime \prime}$ may be chosen as $\Delta_{n+1}$ such that (3) is valid for $i=n$. Let $\Sigma_{n}$ be the $\sigma$-field generated by $\Delta_{n}, \Sigma_{\infty}=\Sigma$, and $f_{\infty}=f$. Then $\left\{f_{n}, \Sigma_{n}, 1 \leqq n \leqq \infty\right\}$ is a martingale such that

$$
\int_{A} f_{n}(s) \mu(d s)=\int_{A} f(s) \mu(d s), \quad A \in \Sigma_{n} .
$$

Therefore by Theorem B,

$$
\lim _{n \rightarrow \infty}\left|f_{n}-f_{\infty}\right|=0,
$$

which contradicts (3), so that $\left\{f_{\Delta}\right\}$ is a generalized Cauchy sequence.

Conversely, let us assume that $\varphi$ is additive and $\left\{f_{\Delta}\right\}$ is a generalized sequence. Then $\left\{f_{\Delta}\right\}$ has a limit $f$ in $L_{1}(S, \Sigma, \mu, \mathscr{X})$, and we shall show that 


$$
\varphi(A)=\int_{A} f(s) \mu(d s) .
$$

It is clear that the map from $L_{1}(S, \Sigma, \mu, \mathscr{X})$ into $\mathscr{X}$ defined by

$$
g \rightarrow \int_{A} g(s) \mu(d s), \quad A \in \Sigma,
$$

is continuous, so that

$$
\int_{A} f(s) \mu(d s)=\lim _{\Delta} \int_{A} f_{\Delta}(s) \mu(d s) .
$$

But for any $\Delta$ containing $A$, that is, any $\Delta \succ\{A, S \backslash A\}$

$$
\int_{A} f_{\Delta}(s) \mu(d s)=\int_{A} \sum_{B \in \Delta} \frac{\varphi(B)}{\mu(B)} \chi(s) \mu(d s)=\sum_{B \in \Delta} \frac{\varphi(B \cap A)}{\mu(B \cap A)} \mu(B \cap A)=\varphi(A) .
$$

Therefore

$$
\varphi(A)=\int_{A} f(s) \mu(d s), \quad A \in \Sigma .
$$

The uniqueness of $f$ is clear, and the last assertion of the theorem is easily verified.

Corollary. In order that there exist a function $f \in L_{1}(S, \Sigma, \mu, \mathscr{X})$ such that

$$
\varphi(A)=\int_{A} f(s) \mu(d s), \quad A \in \Sigma,
$$

the necessary and sufficient condition in Theorem 1 may be replaced by each of the following two conditions:

(i) $\varphi$ is additive and for every increasing sequence $\left\{\Delta_{i}\right\}$ the sequence $\left\{f_{\Delta_{i}}\right\}$ is a Cauchy sequence in $L_{1}(S, \Sigma, \mu, \mathscr{X})$.

(ii) The restriction of $\varphi$ to any $\sigma$-field generated by a countable subsystem of $\Sigma$ may be represented as an indefinite integral with respect to $\mu$.

Proof. (i). The necessity is an immediate consequence of Theorem 1. To prove the sufficiency assume that $\varphi$ is not an indefinite integral. Then according to the necessary condition in Theorem 1, there exists an increasing sequence $\left\{\Delta_{i}\right\}$ and an $\varepsilon_{0}>0$ such that

$$
\left|f_{\Delta_{i}}-f_{\Delta_{i+1}}\right|_{1}>\varepsilon_{0},
$$

which contradicts the assumptions and terminates the proof of (i).

(ii) In view of Theorem B the necessity is obvious. To prove the suf- 
ficiency of the condition, let $\left\{\Delta_{i}\right\}$ be an arbitrary increasing sequence. Obviously, $\Gamma=\bigcup_{i=1}^{\infty} \Delta_{i}$ is countable or finite and generates a $\sigma$-field $\Sigma_{0}$ which fulfills the condition in (ii). Therefore, there exists a function $f_{0} \in L_{1}\left(S, \Sigma_{0}, \mu, \mathscr{X}\right)$ such that

$$
\varphi(A)=\int_{A} f_{0}(s) \mu(d s), \quad A \in \Sigma_{0} .
$$

Then, according to Theorem $\mathrm{B}$, the sequence $\left\{f_{\Delta_{i}}\right\}$ is a Cauchy sequence, and as a consequence of the sufficiency in (i) the existence of a function $f \in L_{1}(S, \Sigma, \mu, \mathscr{X})$ is established such that

$$
\varphi(A)=\int_{A} f(s) \mu(d s), \quad A \in \Sigma,
$$

which terminates the proof of (ii).

\section{3. $\mathscr{X}$-valued martingales and the Radon-Nikodym theorem.}

It is shown by R. S. Phillips [11; Theorems 5, 6] that if $\mathscr{X}$ is a reflexive Banach space, then the following statement is valid:

I. Let $(S, \Sigma, \mu)$ be a $\sigma$-finite measure space, and let $\varphi: \Sigma \rightarrow \mathscr{X}$ be a $\mu$-continuous, $\mathscr{X}$-valued measure of bounded variation.

Then there exists a unique function $f \in L_{1}(S, \Sigma, \mu, \mathscr{X})$ such that

Moreover $v(\varphi, S)=|f|_{1}$.

$$
\varphi(A)=\int_{A} f(s) \mu(d s), \quad A \in \Sigma .
$$

On the other hand S. D. Chatterji [2, Theorem 3,3,2], [4, Theorem 3] has proved the following convergence theorem for any reflexive space $\mathscr{X}$ :

II. Let $(S, \Sigma, \mu)$ be a finite measure space and let $\left\{f_{n}, \Sigma_{n}, 1 \leqq n<\infty\right\}$ be an $\mathscr{X}$-valued martingale in $L_{1}(S, \Sigma, \mu, \mathscr{X})$. Assume that the functions in the sequence $\left\{\left|f_{n}(\cdot)\right|\right\}$ are uniformly integrable.

Then there exists a function $f_{\infty} \in L_{1}(S, \Sigma, \mu, \mathscr{X})$ such that

$$
\lim _{n \rightarrow \infty}\left|f_{n}-f_{\infty}\right|_{1}=0 \text {. }
$$

The following theorem will point out the relation between statements I and II, when $\mathscr{X}$ is an arbitrary Banach space.

Theorem 2. For an arbitrary Banach space $\mathscr{X}$ the statements $I$ and $I I$ are equivalent. 
Proof. Assume that $\mathrm{I}$ is valid and let $\left\{f_{n}, \Sigma_{n}, 1 \leqq n<\infty\right\}$ be an $\mathscr{X}$-valued martingale, where $\left\{\left|f_{n}(\cdot)\right|\right\}$ consists of uniformly integrable functions. Putting

$$
\Gamma=\bigcup_{n=1}^{\infty} \Sigma_{n}
$$

$\Gamma$ becomes a field and we define $\varphi: \Gamma \rightarrow \mathscr{X}$ by

$$
\varphi(A)=\int_{A} f_{n}(s) \mu(d s), \quad A \in \Sigma_{n} .
$$

Obviously, $\varphi$ is an additive $\mathscr{X}$-valued set function, and the uniform integrability insures that $v(\varphi, S)$ is finite and that $\varphi$ is $\mu$-continuous. Consequently, $\varphi$ is countably additive and, according to Theorem A, $\varphi$ can be extended to an $\mathscr{X}$-valued measure of bounded variation. Statement I implies the existence of a function $f \in L_{1}(S, \Sigma, \mu, \mathscr{X})$, such that

$$
\varphi(A)=\int_{A} f(s) \mu(d s), \quad A \in \Sigma .
$$

By Theorem B there exists a unique martingale $\left\{f_{n}{ }^{\prime}, \Sigma_{n}, 1 \leqq n \leqq \infty\right\}$ such that

and

$$
\int_{A} f_{n}{ }^{\prime}(s) \mu(d s)=\int_{A} f(s) \mu(d s), \quad A \in \Sigma_{n},
$$

$$
\lim _{n \rightarrow \infty}\left|f_{n}^{\prime}-f_{\infty}\right|=0 .
$$

Since $f_{n}{ }^{\prime}(\cdot)$ must equal $f_{n}(\cdot)$ a.e., statement II holds.

Conversely, assume that II is valid, and let $\varphi: \Sigma \rightarrow \mathscr{X}$ be a $\mu$-continuous $\mathscr{X}$-valued measure of bounded variation. It is easy to verify that it is sufficient to consider the case where $(S, \Sigma, \mu)$ is finite. By a straight forward application of the Corollary (i) to Theorem 1 it is possible to show that there exists a function $f \in L_{1}(S, \Sigma, \mu, \mathscr{X})$ such that

$$
\varphi(A)=\int_{A} f(s) \mu(d s), \quad A \in \Sigma .
$$

It is known that the classical Radon-Nikodym theorem cannot be extended to the case of a general Banach space. This fact implies, together with Theorem 2 , the existence of an $\mathscr{X}$-valued martingale (for a certain Banach space $\mathscr{X}$ ) satisfying the conditions in II and not being a Cauchy sequence. We shall give a simple example of such a martingale. (An exercise concerning this problem is to be found in [1, exercice 17, p. 92] and another example occurs in Chatterji [3].) 
Example. Let $S$ denote $\{s \mid 0 \leqq s \leqq 1\}, \Sigma$ the Borel field generated by the usual topology on $S$ and let $\mu$ denote the usual Borel measure.

Further let $\mathscr{X}$ be the linear space $c_{0}$, that is, the space of all bounded sequences $x=\left\{\alpha_{n}\right\}$ of scalars converging to zero, which provided with the norm

$$
|x|=\sup _{i}\left|\alpha_{i}\right|
$$

becomes a separable Banach space. Finally, put $e_{n}=\left\{\delta_{i n}\right\}, n=1,2,3, \ldots$, where $\delta_{i n}=0$ for $i \neq n$ and $\delta_{n n}=1$.

For every $n, n=1,2,3, \ldots$, define

$$
A_{i}{ }^{n}=\left\{s \mid(i-1) / 2^{n} \leqq s<i / 2^{n}\right\}, \quad i=1,2,3, \ldots, 2^{n},
$$

and let $\Sigma_{n}$ denote the $\sigma$-field generated by $\left\{A_{i}{ }^{n}\right\}, i=1,2, \ldots, 2^{n}$. Defining a sequence of functions $\left\{f_{n}\right\}$ in the following way:

$$
\begin{aligned}
f_{1}(s) & =(-1)^{i} e_{1}, & & s \in A_{i}{ }^{1}, i=1,2, \\
\vdots & & & \\
f_{n}(s) & =f_{n-1}(s)+(-1)^{i} e_{n}, & & s \in A_{i}{ }^{n}, i=1,2, \ldots, 2^{n},
\end{aligned}
$$

we obtain a martingale $\left\{f_{n}, \Sigma_{n}, 1 \leqq n<\infty\right\}$ satisfying the conditions in statement II, but not being a Cauchy sequence.

\section{REFERENCES}

1. N. Bourbaki, Eléments de mathématique, Livre VI Intégration, Ch. 6 Intégration vectorielle (Act. Sci. Ind. 1281), Paris, 1959.

2. S. D. Chatterji, Martingales of Banach-valued random variables, Thesis, Michigan State University, 1960.

3. S. D. Chatterji, Martingales of Banach-valued random variables, Bull. Amer. Math. Soc. 66 (1960), 395-398.

4. S. D. Chatterji, $A$ note on the convergence of Banach-space valued martingales, Math. Ann. 153 (1964), 142-149.

5. J. L. Doob, Stochastic processes, New York, 1953.

6. M. Driml and O. Hanš, Conditional expectations for generalized random variables, Transactions of the Second Prague Conference on information theory, statistical decision functions and random processes, 1960.

7. N. Dunford and J. T. Schwartz, Linear operators I, New York, 1958.

8. M. Metivier, Limites projectives de mesures. Martingales. Applications, Annali Mat. Pura Appl. (4), 63 (1963), 225-352.

9. M. Metivier, Convergences de martingales à valeurs vectorielles, Bull. Soc. Math. Grèce (Nouvelle serie) 5 (1964), 54-74.

10. J. Neveu, Relation entre la théorie des martingales et la théorie ergodique, Colloque internationale du C. N. R. S. 1964, Théorie du potentiel, Exposé no 12.

11. R. S. Phillips, On weakly compact subsets of a Banach space, Amer. J. Math. 65 (1943), 108-136. 
12. F. S. Scalora, Abstract martingale convergence theorems, Pacific J. Math. 11 (1961), 347-374.

13. Symposium on probability methods in analysis (Lecture Notes in Mathematics 31), Berlin · Heidelberg · New York, 1967.

14. A. I. Tulcea and C. I. Tulcea, Abstract ergodic theorems, Proc. Nat. Acad. Sci. U. S. A. 48 (1962), 204-206.

15. A. I. Tulcea and C. I. Tulcea, Abstract ergodic theorems, Trans. Amer. Math. Soc. 107 (1963), 107-124.

BISP PEDERS VEJ 8, VIRUM, DENMARK 